\title{
Decision-Making Based on Consumers' Perceived Value in Different Remanufacturing Modes
}

\author{
Wei Li, ${ }^{1,2}$ Hang Wu, ${ }^{1}$ and Liurui Deng ${ }^{3}$ \\ ${ }^{1}$ School of Economic and Trade, Hunan University, Changsha 410079, China \\ ${ }^{2}$ Hunan Key Laboratory of Logistics Information and Simulation Technology, Changsha 410079, China \\ ${ }^{3}$ College of Economics and Management, Hunan Normal University, Changsha 410081, China \\ Correspondence should be addressed to Wei Li; liweihncs@hnu.edu.cn
}

Received 6 July 2014; Revised 5 November 2014; Accepted 7 November 2014

Academic Editor: Binggen Zhang

Copyright (C) 2015 Wei Li et al. This is an open access article distributed under the Creative Commons Attribution License, which permits unrestricted use, distribution, and reproduction in any medium, provided the original work is properly cited.

\begin{abstract}
We investigate how the diversity of consumers' perceived value in different remanufacturing modes affects remanufacturing decision-making. We establish a two-stage optimal decision-making model of original equipment manufacturer (OEM) remanufacturing and a noncooperative game model of third party remanufacturer (TPR) remanufacturing and then analyze the optimal decisions of OEM and TPR. Comparing the effects of consumers' perceived value on remanufacturing decision-making in different modes, we find that when OEM remanufactures products, consumers' perceived value has a negative effect on new products' price and quantity and has a positive effect on remanufactured products' quantity and when TPR remanufactures products, consumers' perceived value has a positive effect on new products price and quantity and has a negative effect on remanufactured products' quantity. Compared with OEM remanufacturing, TPR remanufacturing can raise the profits of OEM and whole closed-loop supply chain, but it will lower the quantity of remanufacturing products.
\end{abstract}

\section{Introduction}

With the strengthening of people's environmental awareness, remanufacturing attracts more and more attention. The original equipment manufacturers (OEMs) of electronic products find that recycling and manufacturing of waste products could get a good reputation and a handsome profit for them. Therefore, the electronics' recycling and remanufacturing in closed-loop supply chain have been a hot spot of the relevant business and academia [1].

At present, the product recycling and remanufacturing are a hot topic. There are many researches about inventory control [2], product pricing [3], and coordination in the process of product remanufacturing. In terms of product pricing, $\mathrm{Gu}$ et al. [4] study the waste products pricing strategies in reverse supply chain system made of individual manufacturers and retailers, which is based on game theory. $\mathrm{Xu}$ et al. [5] find out the optimal pricing strategy and corresponding product recovery rate and make comparative analysis with a single-pricing strategy. Recently, some researches focus on dynamic pricing for remanufactured products in multiperiod [6] and uncertain quality [7].

When studying the remanufacturer and manufacturer's optimal pricing decision in cooperation and competitive modes, Kai et al. [8] compare the two models in terms of pricing, profit, sales, and other angles. Considering the coordination of closed-loop supply chain, Ge et al. [9] make analysis of the closed-loop supply chain recovery in different patterns based on game theory and designs income and expense sharing contract to coordinate the closed-loop supply chain under decentralized decision-making. Based on three premises which the government has used to provide incentives and punishments for retailers, Wenwei et al. [10] obtain the optimal retailer's contract provided by the manufacturer in different modes. Jena and Sarmah [11] study cooperation and competition issues in a closed-loop supply chain.

Besides, researches have been done on management efficiency and operational efficiency of the closed-loop supply chain as well. Ferguson and Toktay [12] find that TPR 
threatens the OEM by cannibalization effect, and they compare the two strategies that OEMs use for preventing TPR from entering the market again. Wu et al. [13] analyze the intraindustry competition between retailers and vertical competition between the retailers and suppliers under six different power structures. Almost at the same time, Wu [14] proposes combining the retailers' offer and service as the key factor in the game of the OEM and TPR, then arrives at a balanced state of both profits, and raises the reference for the management of the supply chain parties program based on the theory.

With the development of psychological theory, many literatures gradually consider the consumer psychology as a factor influencing decision-making and analyze the impact of consumer demand on remanufacture decision-making from the market side. Atasu et al. [15] divide the consumers of electronic products into general consumer and green consumer and analyze the influence of the green consumers' market share on remanufacturing decision-making. Oraiopoulos et al. [16] propose "the resale value effect" in their study, which means that consumers will consider a new products' resale value before buying it. The greater the resale value is, the more willing the consumer will be to buy new products. Later, Agrawal et al. [17] prove that the "consumers' perceived value" exists through behavioral experiments; it indicates that the presence of remanufactured goods would impact the consumers' perceived value of new products and then influence the willingness to pay.

Remanufacturing can be undertaken by either OEM or remanufacturer; different remanufacturing modes impact consumers' perceived value of new products in different directions and then affect remanufacturing decisions. Therefore, this paper brings in the "consumers' perceived value effect" and analyzes the optimal decisions in different remanufacturing modes: in closed-loop supply chain in which OEM is dominant, when OEM remanufactures products, its remanufacturing decisions are controlled by direct pricing; when TPR remanufactures products, OEM influences the remanufacturing decisions by charging "patent licensing fees" from TPR [15]. Then we compare the profits of each member in the closed-loop supply chain in different remanufacturing modes. This paper has some important implications for improving remanufacturing decision-making based on nonrational behavior of consumers.

\section{Description of the Problem}

We consider a closed-loop supply chain which has two members; the decision-making process under different remanufacturing patterns is as follows.

In the model of OEM remanufacturing, OEM produces new products in the first stage, and, in the second stage, OEM produces both new products and remanufactured products. In the model of TPR remanufacturing, OEM produces new products during two periods and charges a "patent licensing fee" [18] from the remanufacturer, and TPR produces remanufactured products in the second stage.
The paper has eight basic assumptions as follows.

Assumption 1. The cost of production of new products for OEM is $c$; the cost of the OEM and TPR's remanufacturing (including the recycling and reprocessing), respectively, is the same $c_{r}$, and $c<c_{r}$, because the cost savings are an important driving force for remanufacturing activities [15].

Assumption 2. When TPR remanufactures products, for the purpose of protecting the proprietary technology and controlling remanufacturing decisions, OEM charges the patent relicensing fee $h$ for per unit remanufactured product.

Assumption 3. In the first stage, OEM sells the new products at price $p_{1}$ and the optimal production of the new product is $q_{1}$; and in the second stage, OEM sells the new products at price $p_{n}$ and the optimal production of the new product is $q_{n}$. Likewise, when OEM remanufactures products, the remanufactured products' price is $p_{r}$; and when TPR remanufactures products, its price is $p_{r}+h(h$ is considered as relicensing fee and obtained by the OEM [18], and TPR gets the revenue of per unit product $p_{r}$ ), and the optimal production of the remanufactured products is $q_{r}$.

Assumption 4. When TPR remanufactures products, OEM is the leader of the market; the $p_{1}, p_{n}$, and $h$ are determined based on the principle of profit maximization.

Assumption 5. The consumers' willingness to pay for the new product is $\theta$, which is uniformly distributed between 0 and 1 [19].

Assumption 6. The consumers prefer new products; that is, if the consumer is willing to pay $\theta$ for the new products, his willingness to pay for the remanufactured product is just $\rho \theta$ $(0<\rho<1)[12]$.

Assumption 7. Remanufacturing impacts the consumers' perceived value of new products and then impacts the consumer's willingness to pay [13]. To be specific, (1) OEM's remanufacturing reduces consumers' perceived value of new products, and then the willingness to pay is only $\alpha \theta$, assuming that the consumers' perceived value factor is $\alpha$, and $\rho<\alpha \leq$ 1 (although OEM remanufacturing could reduce consumer preference of new products, the consumers' willingness to pay for new products is still greater than the willingness to buy remanufactured products; namely, $0<\rho<\alpha \leq 1$ ); (2) TPR's remanufacturing increases consumers' perceived value of new products; consumers are willing to pay $\beta \theta$ for the new product. Assume that the consumers' perceived value factor is $\beta$, and $\beta \geq 1$. The larger the consumers' perceived value effect, the greater the impact of the remanufacturing activities on consumers' perceived value.

Assumption 8. The market share of first stage and second stage is 1 , respectively, and, in the second stage's remanufacturing activities, the number of the old products' recycling is not restricted [12]. 


\section{The Basic Model}

Firstly, we analyze the pricing process in different remanufacturing modes: when OEM remanufactures products, we study the optimization decision for only one subject; when TPR remanufactures products, we study a noncooperative game problem. Next, we establish the remanufacturing decision model of electronic products which considers the consumers' perceived value effect.

3.1. The Consumer's Utility Function and Production Demand. In the first stage, there are no remanufactured products; consumers only buy new products. Every consumer's utility of buying new products is $U_{1}=\theta-p_{1}$. The demand of the products is $q_{1}=1-p_{1}$ [13].

In the second stage, we discuss two cases in which remanufacturing is undertaken by OEM and TPR, respectively.

(1) OEM Remanufacturing. As OEM remanufacturing will reduce the consumers' perceived value of new products and then reduce the willingness to pay, the consumer's utility of buying the new products is $U_{n}=\alpha \theta-p_{n}(\rho<\alpha \leq 1)$; and the utility of buying remanufactured products is $U_{r}=$ $\rho \theta-p_{r}(0<\rho<1)$. In order to ensure $q_{n}>0$, we have another assumption $\rho \leq \alpha-\left(p_{n}-p_{r}\right)[13]$.

As the consumers will choose the product which makes their own utility maximum, we assume that the set of consumers' purchasing new products is $\Theta_{n}$, and $\Theta_{n}=\{\theta$ : $\left.U_{n} \geq \max \left\{U_{r}, 0\right\}\right\}$; assume the set of consumers' purchasing remanufactured products is $\Theta_{r}$, and $\Theta_{r}=\left\{\theta: U_{r} \geq\right.$ $\left.\max \left\{U_{n}, 0\right\}\right\}$. The new products get a market share of $m_{n}=$ $\int_{\theta \in \Theta_{n}} f(\theta) d \theta$ and the remanufactured products get a market share of $m_{r}=\int_{\theta \in \Theta_{r}} f(\theta) d \theta$.

If $\alpha p_{r} \leq \rho p_{n}$, the consumers' demand of new products is $q_{n}=1 * m_{n}=\left(\alpha-\rho-p_{n}+p_{r}\right) /(\alpha-\rho)$; and the demand of remanufactured products is $q_{r}=1 * m_{r}=\left(\rho p_{n}-\alpha p_{r}\right) / \rho(\alpha-\rho)$.

If $\alpha p_{r}>\rho p_{n}$, the consumers' demand of new products is $q_{n}^{\circ}=(\alpha-\rho) / \alpha$; and the demand of remanufactured products is $q_{r}^{\circ}=0$.

(2) TPR Remanufacturing. As TPR remanufacturing will increase the consumers' perceived value of new products and then improve the willingness to pay, the consumer's utility of buying the new products is $U_{n}=\beta \theta-p_{n}(\beta \geq 1)$; and the utility of buying remanufactured products is $U_{r}=\rho \theta-\left(p_{r}+\right.$ $h)$. In order to ensure $q_{n}>0$, we have another assumption $\rho \leq \beta-\left(p_{n}-p_{r}-h\right)$.

If $\beta\left(p_{r}+h\right) \leq \rho p_{n}$, the consumers' demand of the new products is $q_{n}=1 * m_{n}=\left(\beta-\rho-p_{n}+p_{r}+h\right) /(\beta-\rho)$; and the demand of remanufactured products is $q_{r}=1 * m_{r}=$ $\left(\rho p_{n}-\beta p_{r}-\beta h\right) / \rho(\beta-\rho)$.

If $\beta\left(p_{r}+h\right)>\rho p_{n}$, the consumers' demand of new products is $q_{n}^{\circ}=\left(\beta-p_{n}\right) / \beta$; and the demand of remanufactured products is $q_{r}^{\circ}=0$.

\subsection{The Function of Profit}

(1) OEM Remanufacturing. As previously mentioned, only when $\alpha p_{r} \leq \rho p_{n}$ and $q_{r} \geq 0$, there are remanufacturing activities. So we only consider the OEM's optimal decision in this case. And the profit of the OEM is

$$
\begin{array}{ll}
\prod_{p_{1}, p_{n}, p_{r}}^{\max }=\left(p_{1}-c\right) q_{1}+\left(p_{n}-c\right) q_{n}+\left(p_{r}-c_{r}\right) q_{r} \\
\text { s.t. } \quad p_{1}, p_{n}, p_{r} \geq 0 \\
\quad p_{1}, p_{n} \geq c \\
\quad p_{r} \geq c_{r} .
\end{array}
$$

(2) TPR Remanufacturing. Similarly, in order to ensure the existence of the remanufacturing activities, we just consider the case of $\beta\left(p_{r}+h\right) \leq \rho p_{n}$.

The OEM's profit is

$$
\begin{aligned}
& \prod_{p_{1}, p_{n}, h}^{\max }=\left(p_{1}-c\right) q_{1}+\left(p_{n}-c\right) q_{n}+h q_{r} \\
& \text { s.t. } \quad p_{1}, p_{n}, h \geq 0 \\
& \quad p_{1}, p_{n} \geq c .
\end{aligned}
$$

TPR's profit is

$$
\begin{aligned}
\prod_{p_{r}}^{\max }= & \left(p_{r}-c_{r}\right) q_{r}-h q_{r} \\
\text { s.t. } & p_{r} \geq c_{r} \\
& h>0 .
\end{aligned}
$$

\section{Remanufacturing Decisions Based on Consumers' Perceived Value}

For convenience, when OEM remanufactures products, we denote the OEM's profit as $\prod_{1 m}$; and when TPR remanufactures products, we denote TPR's profit as $\prod_{t}$ and OEM's profit $\prod_{2 m}$. Besides, the profit of the whole supply chain is $\prod_{1}$ when OEM remanufactures products; and the profit of the whole products is $\prod_{2}$ when TPR remanufactures products.

In the next part, we have the equilibrium analysis in the two modes, respectively.

4.1. OEM Remanufacturing. In this case, OEM is the only manufacturer, which produces new products in the first stage and both new products and remanufactured products at the same time in the second stage. According to the profit function $\prod_{1 m}=\left(p_{1}-c\right) q_{1}+\left(p_{n}-c\right) q_{n}+\left(p_{r}-c_{r}\right) q_{r}$, one has the following.

Proposition 9. when OEM remanufactures products, if the consumers' perceived value coefficient $\alpha$ meets $\rho+\left(c-c_{r}\right)<$ $\alpha<c \rho / c_{r}$, then the OEM's balance set of decision-making is $p_{1}^{*}, p_{n}^{*}, p_{r}^{*}, q_{1}^{*}, q_{n}^{*}, q_{r}^{*}$. To be more specific,

$$
p_{n}^{*}=\frac{\alpha+c}{2},
$$




$$
\begin{aligned}
& p_{r}^{*}=\frac{\rho+c_{r}}{2}, \\
& q_{n}^{*}=\frac{\alpha+c_{r}-\rho-c}{2(\alpha-\rho)}, \\
& q_{r}^{*}=\frac{c \rho-\alpha c_{r}}{2 \rho(\alpha-\rho)} .
\end{aligned}
$$

Proof. When OEM remanufactures products, according to the profit function $\prod_{1 m}$, we can obtain the optimal prices of new products and remanufactured products as $p_{n}^{*}$ and $p_{r}^{*}$. And then we can also obtain the optimal quantity as $q_{n}^{*}$ and $q_{r}^{*}$. In order to make remanufacturing activity possible, the following conditions must be met:

$$
\begin{aligned}
& \text { from } p_{n}^{*}>c \text { : we know } \alpha>c \text {; } \\
& \text { from } p_{r}^{*}>c_{r} \text { : we know that } \rho>c_{r} \\
& \text { from } q_{n}^{*}>0 \text { : we know } \alpha-\rho>c-c_{r}>0 \text {; } \\
& \text { from } q_{r}^{*}>0 \quad \text { we know: } \alpha c_{r}-\rho c<0
\end{aligned}
$$
proven.

According to (1), (2), (3), and (4), Proposition 9 can be

Conclusion 1. Consumers' perceived value coefficient impacts the optimal price of new products in the second stage and does not impact the optimal remanufacturing product price; the larger the $\alpha$ is, the higher the $p_{n}^{*}$ is, and at the time $\alpha=1$, $p_{n}^{*}$ takes the maximum value.

Conclusion 1 shows that when OEM remanufactures products, the optimal price of new products in the second stage increases with the increase of the coefficient of consumers' perceived value; that is, OEM remanufacturing has negative effects on the optimal price of new products.

Conclusion 2. In the second stage, the optimal quantity of new product will increase as $\alpha$ increases; the optimal quantity of remanufactured product will decrease as $\alpha$ increases.

Conclusion 2 shows that when OEM remanufactures products, the optimal quantity of new products in the second stage will decrease as the coefficient of consumers' perceived value increases; namely, OEM remanufacturing has negative effects on the optimal quantity of new products. The optimal quantity of remanufactured products will increase as the coefficient of consumers' perceived value increases, which means that OEM remanufacturing has positive effects on the optimal quantity of remanufactured products.

Conclusion 3. The OEM's profit will increase as the $\alpha$ increases.

Conclusion 3 shows that when OEM remanufactures products, if consumers' perceived value effect is smaller, that is to say, $\alpha$ is larger, OEM can gain more profit and less vice versa. It means that OEM remanufacturing has negative effects on its profit. It explains why most of the international famous electronic OEMs do not remanufacture products themselves.

4.2. TPR Remanufacturing. In this case, the OEM produces new products both in the first stage and in the second stage; TPR produces remanufactured products.

Proposition 10. When TPR remanufactures products, the supply chain balanced decision set is $\left(p_{1}^{* *}, p_{n}^{* *}, p_{r}^{* *}, q_{1}^{* *}, q_{n}^{* *}\right.$, $\left.q_{r}^{* *}\right)$, in which $p_{r}^{* *}$ is defined as "unit remanufactured product revenue of TPR." Each state variable is as follows:

$$
\begin{aligned}
p_{n}^{* *}= & \frac{2 \beta^{2}-2 \beta \rho+2 \beta h+c_{r} \beta+2 c \beta-\rho c}{4 \beta-2 \rho}, \\
p_{r}^{* *}= & \frac{\left(\beta^{2}-\beta \rho+\beta h+(1 / 2) c_{r} \beta+c \beta-(1 / 2) \rho c\right) \rho}{(4 \beta-2 \rho) \beta} \\
& -\frac{1}{2} h+\frac{1}{2} c_{r}, \\
q_{n}^{* *}= & \frac{2 \beta^{2}-2 c \beta-2 \beta \rho+c_{r} \beta+\rho c}{4 \beta(\beta-\rho)}, \\
q_{r}^{* *}= & \frac{\beta^{2}-\beta \rho+\beta h+(1 / 2) c_{r} \beta+c \beta-(1 / 2) \rho c}{2(2 \beta-\rho)(\beta-\rho)} \\
& -\frac{\beta h+c_{r} \beta}{2 \rho(\beta-\rho)} .
\end{aligned}
$$

Conclusion 4. The price of new products $p_{n}^{* *}$ in the second stage will increase as the consumers' perceived value coefficient $\beta$ increases.

Conclusion 4 shows that when TPR remanufactures products, the consumers' perceived value for the new product is influenced by the behavior of TPR's remanufacturing; TPR remanufacturing has positive effects on the optimal price of new products. And the optimal price increases as the perceived value coefficient increases.

Conclusion 5. The optimal quantity of new products $q_{n}^{* *}$ in the second stage will increase as the $\beta$ increases. The optimal quantity $q_{r}^{* *}$ for TPR remanufacturing will decline as $\beta$ increases.

Conclusion 5 shows that when TPR remanufactures products, TPR remanufacturing has positive effects on the optimal quantity of new products. The optimal quantity of remanufactured products will increase with the decrease of the coefficient of consumers' perceived value, which means that TPR remanufacturing has negative effects on the optimal quantity of remanufactured products.

\subsection{Profit Comparison in Different Remanufacturing Mode}

Proposition 11. $m_{1}$ is defined as OEM profit difference under two cases (OEM remanufacturing and TPR remanufacturing), assuming that $m_{1}=\prod_{2 m}-\prod_{1 m} ; m_{2}$ is defined as the 
OEM's profit gap when $c=0.4, c_{r}=0.2, \rho=0.6$, and $h=0.15$

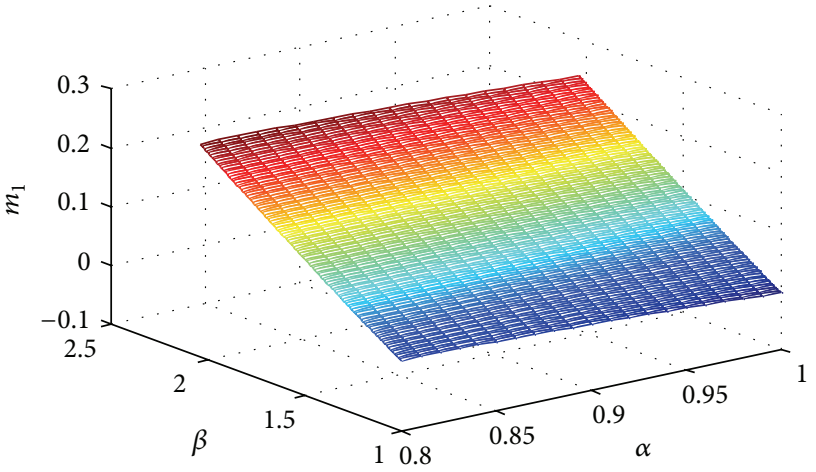

FIgURE 1: Consumers' perceived value impact on $m_{1}^{*}$.

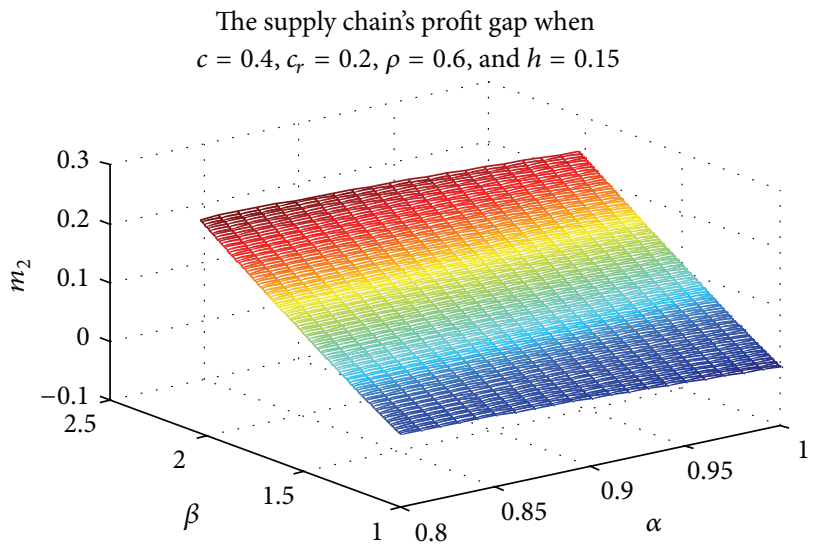

FIGURE 2: Consumers' perceived value impact on $m_{2}^{*}$.

closed-loop supply chain profit difference between the two cases, assuming that $m_{2}=\prod_{2}-\prod_{1}$. We can obtain the optimal profit differences in different remanufacturing modes.

Therefore, we can work out the optimal profit differences $m_{1}^{*}=\prod_{2 m}^{*}-\prod_{1 m}^{*}$ and $m_{2}^{*}=\prod_{2}^{*}-\prod_{1}^{*}$, the optimal price $\Delta p_{n}=$ $p_{n}^{* *}-p_{n}^{*}, \Delta p_{r}=p_{r}^{* *}-p_{r}^{*}$, and optimal quantity differences $\Delta q_{n}=q_{n}^{* *}-q_{n}^{*}, \Delta q_{r}=q_{r}^{* * *}-q_{r}^{*}$ in different remanufacturing modes.

Due to the complexity of these functions, we will analyze how the consumers' perceived value impacts the related profit, price, and quantity with numerical simulation in the next part.

\section{Numerical Simulation}

Because the final profit functions are so complex that it is difficult to compare them under the two different remanufacturing modes, we do further research by numerical analysis. Assuming that $c=0.4, c_{r}=0.2, h=0.15$, and $0.8<$ $\alpha<1,1<\beta<2.0484$, we can discuss the profit, price, and quantity differences between OEM remanufacturing and TPR remanufacturing modes by Figures 1-6.

It can be seen from Figure 1 that when OEM remanufactures products, OEM's profit will decrease as $\alpha$ increases; when TPR remanufactures products, OEM's profit

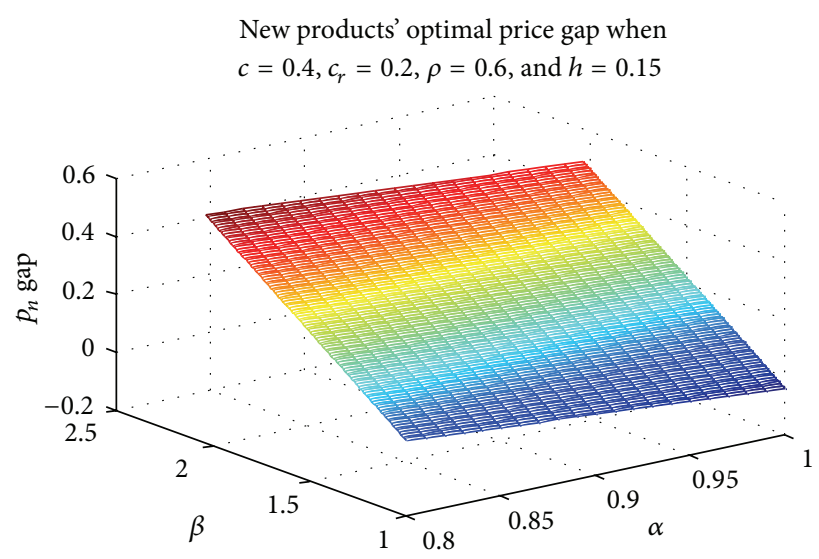

FIgURE 3: Consumers' perceived value impact on $\Delta p_{n}$.

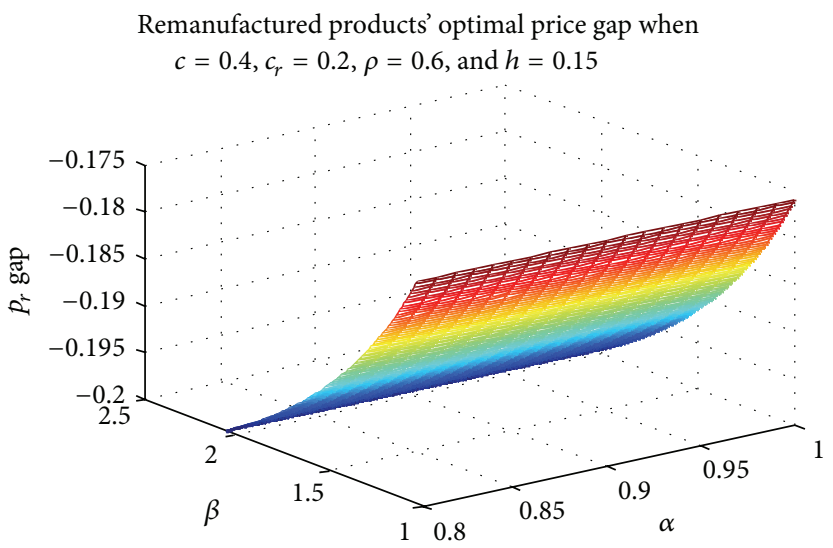

FIGURE 4: Consumers' perceived value impact on $\Delta p_{r}$.

will increase as $\beta$ increases. In other words, in either remanufacturing modes, the greater the consumers' perceived value effect is, the more the OEM's profit will be.

It can be seen from Figure 2 that when OEM remanufactures products, the whole closed-loop supply chain's profit will decrease as $\alpha$ increases; when TPR remanufactures products, the whole closed-loop supply chain's profit will increase as $\beta$ increases. To put it in another way, in either remanufacturing modes, the greater the consumers' perceived value effect is, the more the whole closed-loop supply chain profit will be.

It can be seen from Figure 3 that when OEM remanufactures products, new product price will decrease as $\alpha$ increases; when TPR remanufactures products, new product price will increase as $\beta$ increases. In other words, in either remanufacturing modes, the greater the consumers' perceived value effect is, the higher the new product price will be.

It can be seen from Figure 4 that when OEM remanufactures products, remanufactured product price will decrease as $\alpha$ increases; when TPR remanufactures products, there will be $\beta^{\prime}$, and when $\beta<\beta^{\prime}$, remanufactured product price will decrease as $\beta$ increases; when $\beta \geq \beta^{\prime}$ remanufactured product price will increase as $\beta$ increases. 


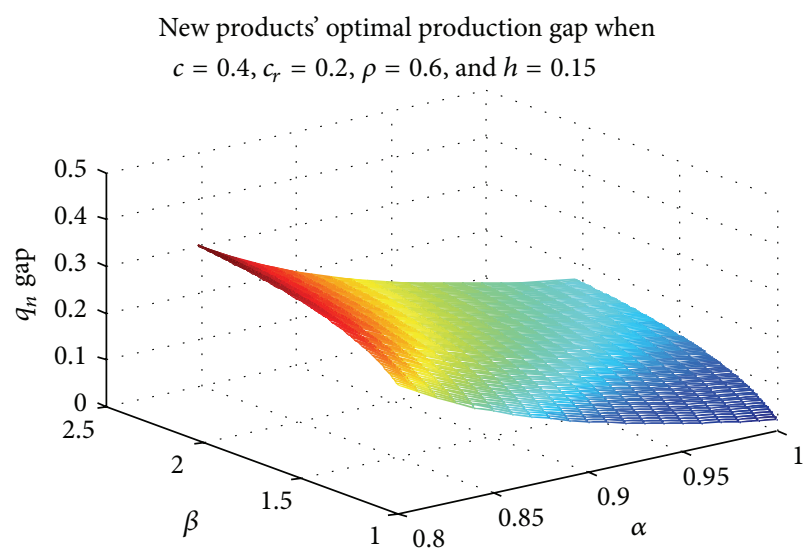

FIgURE 5: Consumers' perceived value impact on $\Delta q_{n}$.

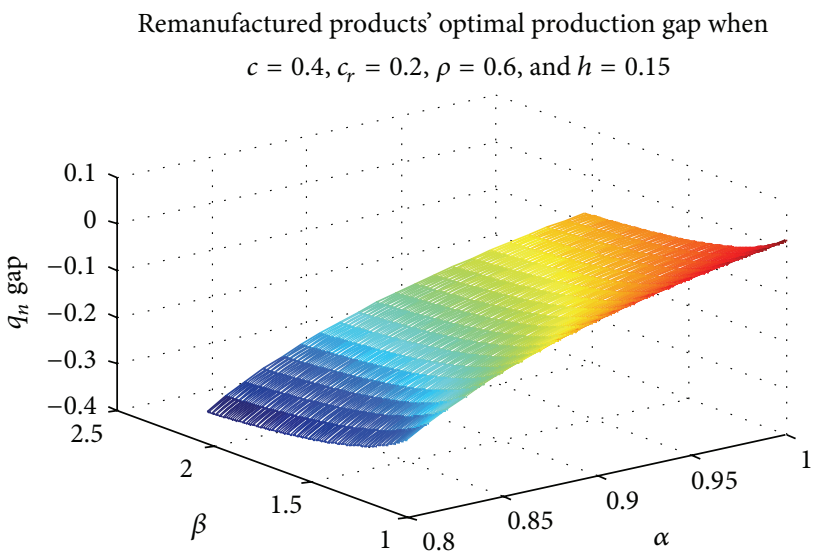

FIGURE 6: Consumers' perceived value impact on $\Delta q_{r}$.

It can be seen from Figure 5 that when OEM remanufactures products, the new products quantity will increase as $\alpha$ increases; when TPR remanufactures products, the new products quantity will increase as $\beta$ increases.

It can be seen from Figure 6 that when OEM remanufactures products, the remanufactured products quantity will decrease as $\alpha$ increases; when TPR remanufactures products, the remanufactured products quantity will decrease as $\beta$ increases.

\section{Conclusions}

In this paper, we study the impacts of consumers' perceived value on the decision-making process; and we also compare the optimal decisions in different remanufacturing modes. By analyzing the impacts of consumers' perceived value on the remanufacturing activities, we can draw the conclusions as follows. (1) Considering the consumers' perceived value, OEM's remanufacturing has negative effects on the price and quantity of new products and has positive effects on the quantity of remanufactured product and negative effects on OEM's profits. (2) TPR's remanufacturing has positive effects on the optimal prices of new products and positive effects on the optimal quantity of new products and also has negative effects on the optimal quantity of remanufactured product. (3) Considering the impacts of consumers' perceived value effect, TPR's remanufacturing can make OEM and the whole closed-loop supply chain get more profits.

The core idea of this study is the consumer psychological factors' influence on remanufacturing activities. In the future, we can extend the closed-loop supply chain research vertically and horizontally: on the one hand, considering the closed-loop supply chain by horizontal competition, we can analyze the consumers' perceived value's impacts on remanufacturing decisions made of one OEM and multiple TPR manufacturers. On the other hand, considering the closed-loop supply chain by vertical competition, we can analyze impacts of consumers' perceived value on remanufacturing decisions made of one OEM and multiple recyclers in multistage games.

\section{Appendix}

In order to make the remanufacturing activities possible, it must meet the following conditions.

(1) OEM Remanufacturing. Consider

$$
\begin{aligned}
& \text { For } p_{n}^{*}>c, \text { then } \alpha>c ; \\
& \text { For } p_{r}^{*}>c_{r}, \quad \text { then } \rho>c_{r} ; \\
& \text { For } q_{n}^{*}>0, \quad \text { then } \alpha-\rho>c-c_{r}>0 ; \\
& \text { For } q_{r}^{*}>0, \quad \text { then } \alpha c_{r}-\rho c<0 .
\end{aligned}
$$

(2) TPR Remanufacturing. Consider

$$
\begin{aligned}
& \text { For } p_{n}^{* *}>c, \\
& \text { then } 2 \beta^{2}-2 \beta \rho+2 \beta h+c_{r} \beta-2 c \beta+\rho c>0 \text {; } \\
& \text { For } p_{r}^{* *}>c_{r}, \\
& \text { then } 2 \rho \beta^{2}-2 \beta \rho^{2}+4 \rho \beta h+3 \rho c_{r} \beta+2 \rho c \beta \\
& \qquad-\rho^{2} c-4 \beta^{2} h-4 c_{r} \beta^{2}>0 \text {; } \\
& \text { For } q_{n}^{* *}>0 \\
& \text { then } 2 \beta^{2}-2 c \beta-2 \beta \rho+c_{r} \beta+\rho c>0 ; \\
& \text { For } q_{r}^{* *}>0, \\
& \text { then } 2 \rho \beta^{2}-2 \beta \rho^{2}+4 \rho \beta h+3 \rho c_{r} \beta+2 \rho c \beta \\
& \quad-\rho^{2} c-4 \beta^{2} h-4 c_{r} \beta^{2}>0 .
\end{aligned}
$$


Proof of Conclusion 1. The optimal price of new product $p_{n}^{*}=$ $(\alpha+c) / 2$ and the optimal price of remanufactured product $p_{r}^{*}=\left(\rho+c_{r}\right) / 2$ make the first order to $\alpha$; the results are $1 / 2$ and 0 .

The conclusion is proven.

Proof of Conclusion 2. The optimal quantity of new products $q_{n}^{*}=0.5\left(\alpha+c_{r}-\rho-c\right) /(\alpha-\rho)$ makes the first order to $\alpha$; we get $0.5\left(c-c_{r}\right) /(\alpha-\rho)^{2}>0$; the optimal quantity of remanufactured products $q_{r}^{*}=0.5\left(c \rho-\alpha c_{r}\right) / \rho(\alpha-\rho)$ makes the first order to $\alpha$; we get $-0.5\left(c-c_{r}\right) /(\alpha-\rho)^{2}<0$.

Then, the conclusion is proven.

Proof of Conclusion 3. $\prod_{1} m=(-(1 / 2) c+1 / 2)^{2}+((1 / 2) \alpha-$ $(1 / 2) c)\left((1 / 2) \alpha-(1 / 2) \rho-(1 / 2) c+(1 / 2) c_{r}\right) /(\alpha-\rho)+((1 / 2) \rho-$ $\left.(1 / 2) c_{r}\right)\left((1 / 2)\left(\rho c-\alpha c_{r}\right)\right) / \rho /(\alpha-\rho), \prod_{1} m$ makes the first order to $\alpha$; we get $(1 / 4)\left((\alpha-\rho)^{2}-\left(c-c_{r}\right)^{2}\right) /(\alpha-\rho)^{2}$.

By (A.3), we know $\alpha-\rho>c-c_{r}>0$; then, $(1 / 4)((\alpha-$ $\left.\rho)^{2}-\left(c-c_{r}\right)^{2}\right) /(\alpha-\rho)^{2}>0$.

The conclusion is proven.

Proof of Conclusion 4. In TPR remanufacturing, $p_{n}^{* *}$ makes the first order to $\beta$; we get

$$
\frac{(1 / 2)\left(4 \beta^{2}-4 \beta \rho+2 \rho^{2}-2 \rho h-\rho c_{r}\right)}{(2 \beta-\rho)^{2}} .
$$

$\left(\partial(1 / 2)\left(4 \beta^{2}-4 \beta \rho+2 \rho^{2}-2 \rho h-\rho c_{r}\right) /(2 \beta-\rho)^{2}\right) \times(\partial h)^{-1}=$ $-2 \rho /(2 \beta-\rho)^{2}<0$; we know $h>-(1 / 2)\left(2 \beta^{2}-2 \beta \rho+c_{r} \beta-\right.$ $2 c \beta+\rho c) / \beta$. By (A.6), we know

$$
h<\frac{2 \rho \beta^{2}-2 \beta \rho^{2}+3 \rho c_{r} \beta+2 \rho c \beta-\rho^{2} c-4 c_{r} \beta^{2}}{4 \beta(\beta-\rho)} .
$$

Then, one has the following.

When $h$ equals the minimum value $-(1 / 2)\left(2 \beta^{2}-2 \beta \rho+\right.$ $\left.c_{r} \beta-2 c \beta+\rho c\right) / \beta, x$ reaches the maximum value

$$
\frac{\left(-2 \rho \beta^{2}+4 \beta^{3}-2 \rho c \beta+\rho^{2} c\right)}{\beta}>0 .
$$

When $h$ equals the maximum value $(1 / 4)\left(2 \rho \beta^{2}-2 \beta \rho^{2}+\right.$ $\left.3 \rho c_{r} \beta+2 \rho c \beta-\rho^{2} c-4 c_{r} \beta^{2}\right) / \beta /(-\rho+\beta), x$ reaches the minimum value $(1 / 2)(2 \beta-\rho)\left(4 \beta^{3}-4 \rho \beta^{2}+2 \beta \rho^{2}+\rho c_{r} \beta+2 \rho \beta^{2}-\right.$ $\left.\rho^{2} c\right) / \beta /(-\rho+\beta)>0$; so, the result of the first order to $\beta$ is bigger than 0 .

The conclusion is proven.

Proof of Conclusion 5. (1) $q_{n}^{* *}$ makes the first order to $\beta$; the result is $(1 / 4)\left(-2 \rho c \beta+2 c \beta^{2}-c_{r} \beta^{2}+\rho^{2} c\right) / \beta^{2} /(-\rho+\beta)^{2}$.

Order $x=-2 \rho c \beta+2 c \beta^{2}-c_{r} \beta^{2}+\rho^{2} c$ makes the first order to $\rho$; the result is $-2 c \beta+2 \rho c<0$, when $\rho=0$; $x$ equals the minimum value $2 c \beta^{2}-c_{r} \beta^{2}>0$.

Then, we get $x>0$; the result of the first order to $\beta$ is bigger than 0 .

(2) $q_{r}^{* *}$ makes the first order to $\beta$; the result is

$$
\begin{aligned}
& \frac{2 \rho \beta^{2}-4 \beta \rho^{2}+2 \rho^{3}+8 \rho \beta h-4 \beta^{2} h-4 \rho^{2} h+8 \rho c_{r} \beta-6 c_{r} \beta^{2}-3 \rho^{2} c_{r}+4 c \beta^{2}-4 \rho c \beta+\rho^{2} c}{-4(2 \beta-\rho)^{2}(-\rho+\beta)^{2}} . \\
& \left(\partial\left(\frac{2 \rho \beta^{2}-4 \beta \rho^{2}+2 \rho^{3}+8 \rho \beta h-4 \beta^{2} h-4 \rho^{2} h+8 \rho c_{r} \beta-6 c_{r} \beta^{2}-3 \rho^{2} c_{r}+4 c \beta^{2}-4 \rho c \beta+\rho^{2} c}{-4(2 \beta-\rho)^{2}(-\rho+\beta)^{2}}\right)\right) \\
& \quad \times(\partial h)^{-1}=-\frac{1}{(2 \beta-\rho)^{2}}<0 .
\end{aligned}
$$

When $h$ equals the minimum value $=-(1 / 2)\left(2 \beta^{2}-2 \beta \rho+\right.$ $\left.c_{r} \beta-2 c \beta+\rho c\right) / \beta, x$ equals the minimum value $(2 \beta-\rho)\left(2 \beta^{3}-\right.$ $\left.2 c_{r} \beta^{2}-4 \rho \beta^{2}+\rho c_{r} \beta+3 \rho c \beta+2 \beta \rho^{2}-2 \rho^{2} c\right) / \beta$.

Order $y=2 \beta^{3}-2 c_{r} \beta^{2}-4 \rho \beta^{2}+\rho c_{r} \beta+3 \rho c \beta+2 \beta \rho^{2}-2 \rho^{2} c=$ $(2 \beta-2 c) \rho^{2}+\left(-4 \beta^{2}+c_{r} \beta+3 c \beta\right) \rho+2 \beta^{3}-2 c_{r} \beta^{2}$; through this equation, we know $b^{2}-4 a c=-\beta^{2}\left(-c_{r}+c\right)\left(-9 c+8 \beta+c_{r}\right)<0$.

Namely, the minimum value of opening up parabolic function is greater than zero; the minimum value of $y$ is greater than 0 . The maximum value of $x$ is also greater than 0 .

When $h$ equals the maximum value $(1 / 4)\left(2 \rho \beta^{2}-2 \beta \rho^{2}+\right.$ $\left.3 \rho c_{r} \beta+2 \rho c \beta-\rho^{2} c-4 c_{r} \beta^{2}\right) / \beta /(-\rho+\beta), x$ equals the minimum value $(2 \beta-\rho)\left(-c_{r} \beta^{2}+2 c \beta^{2}-2 \rho c \beta+\rho^{2} c\right) / \beta$.

Order $y=-c_{r} \beta^{2}+2 c \beta^{2}-2 \rho c \beta+\rho^{2} c=\left(2 c-c_{r}\right) \beta^{2}-2 \rho c \beta+$ $\rho^{2} c$; through this equation, we know $b^{2}-4 a c=-4 \rho^{2} c\left(-c_{r}+\right.$ c) $<0$, the minimum value of $y$ is greater than 0 , and the maximum value of $x$ is also greater than 0 .

Then, the result of $q_{r}^{* *}$ 's first order to $\beta$ is smaller than 0 . The conclusion is proven.

\section{Conflict of Interests}

The authors declare that there is no conflict of interests regarding the publication of this paper.

\section{Acknowledgments}

The authors are grateful to the two anonymous referees and the editor for their contribution in significantly improving the final paper. The study is supported by the Philosophy Social Science Foundation of Hunan Province (2010YBA048), the 
Foundation of Ministry of Education on Humanities and Social Science Research (11YJC790084), the Doctoral Program of Higher Education (20110161120032), the National Natural Science Foundation of China (Grant no. 71201051), the Doctor Research Fund (Grant no. 2014BQ11), and Young Talents Training Plan of Hunan Normal University (Grant no. 2014YX04).

\section{References}

[1] P. de Giovanni and G. Zaccour, "A two-period game of a closedloop supply chain," European Journal of Operational Research, vol. 232, no. 1, pp. 22-40, 2014.

[2] X. Sun, M. Wu, and F. Hu, "Two-period inventory control with manufacturing and remanufacturing under return compensation policy," Discrete Dynamics in Nature and Society, vol. 2013, Article ID 871286, 8 pages, 2013.

[3] J. Zhao, W. Liu, and J. Wei, "Pricing and remanufacturing decisions of a decentralized fuzzy supply chain," Discrete Dynamics in Nature and Society, vol. 2013, Article ID 986704, 10 pages, 2013.

[4] Q.-L. Gu, T.-G. Gao, and L.-S. Shi, "Price decision analysis for reverse supply chain based on game theory," System Engineering Theory and Practice, vol. 25, no. 3, pp. 20-25, 2005.

[5] F. Xu, Z. Sheng, and G. Chen, "The remanufactured products pricing strategy in a heterogeneous market," Chinese Journal of Management Science, vol. 16, no. 6, pp. 130-136, 2008.

[6] J.-M. Chen and C.-I. Chang, "Dynamic pricing for new and remanufactured products in a closed-loop supply chain," International Journal of Production Economics, vol. 146, no. 1, pp.153160, 2013.

[7] Y. Xiong, G. Li, Y. Zhou, K. Fernandes, R. Harrison, and Z. Xiong, "Dynamic pricing models for used products in remanufacturing with lost-sales and uncertain quality," International Journal of Production Economics, vol. 147, pp. 678-688, 2014.

[8] W. Kai, X. Zhongkai, and X. Yu, "The research on the model that the remanufacturer collaborates with manufacturer in the some channel," Chinese Journal of Management Science, vol. 20, no. 1, pp. 145-151, 2012.

[9] J. Ge, P. Huang, and J. Li, "Social environmental consciousness and price decision analysis for closed-loop supply chainsbased on vertical differentiation model," Industrial Engineering and Management, no. 4, pp. 6-10, 2007.

[10] G. Wenwei, L. Hu, and M. Qiang, "The contract design of reverse supply chain under the government guidance," Operations Research and Management Science, vol. 21, no. 3, pp. 242249, 2012.

[11] S. K. Jena and S. P. Sarmah, "Price competition and co-operation in a duopoly closed-loop supply chain," International Journal of Production Economics, vol. 156, pp. 246-360, 2014.

[12] M. E. Ferguson and L. B. Toktay, "The effect of competition on recovery strategies," Production and Operations Management, vol. 15, no. 3, pp. 351-368, 2006.

[13] C.-H. Wu, C.-W. Chen, and C.-C. Hsieh, "Competitive pricing decisions in a two-echelon supply chain with horizontal and vertical competition," International Journal of Production Economics, vol. 135, no. 1, pp. 265-274, 2012.

[14] C.-H. Wu, "Product-design and pricing strategies with remanufacturing," European Journal of Operational Research, vol. 222, no. 2, pp. 204-215, 2012.
[15] A. Atasu, M. Sarvary, and L. N. V. Wassenhove, "Remanufacturing as a marketing strategy," Management Science, vol. 54, no. 10, pp. 1731-1746, 2008.

[16] N. Oraiopoulos, M. E. Ferguson, and L. B. Toktay, "Relicensing as a secondary market strategy," Management Science, vol. 58, no. 5, pp. 1022-1037, 2012.

[17] V. V. Agrawal, A. Atasu, and K. van Ittersum, "Remanufacturing, third-party competition, and consumers' perceived value of new products," Working Paper, Georgetown University McDonough School of Business, 2012.

[18] X. Zhongkai, S. Chengran, and P. Zhiqiang, "Closed-loop supply chain coordination research with remanufacturing under patent protection," Journal of Management Science in China, vol. 14, no. 6, pp. 76-85, 2011.

[19] G. Ferrer and J. M. Swaminathan, "Managing new and differentiated remanufactured products," European Journal of Operational Research, vol. 203, no. 2, pp. 370-379, 2010. 


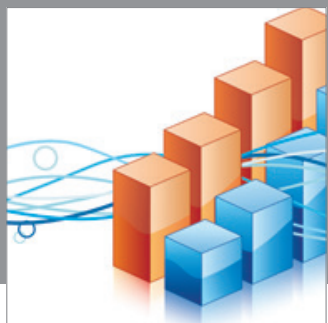

Advances in

Operations Research

mansans

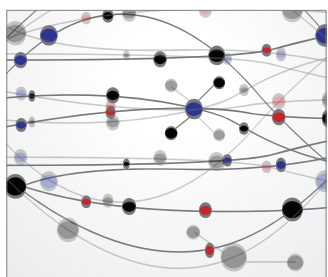

The Scientific World Journal
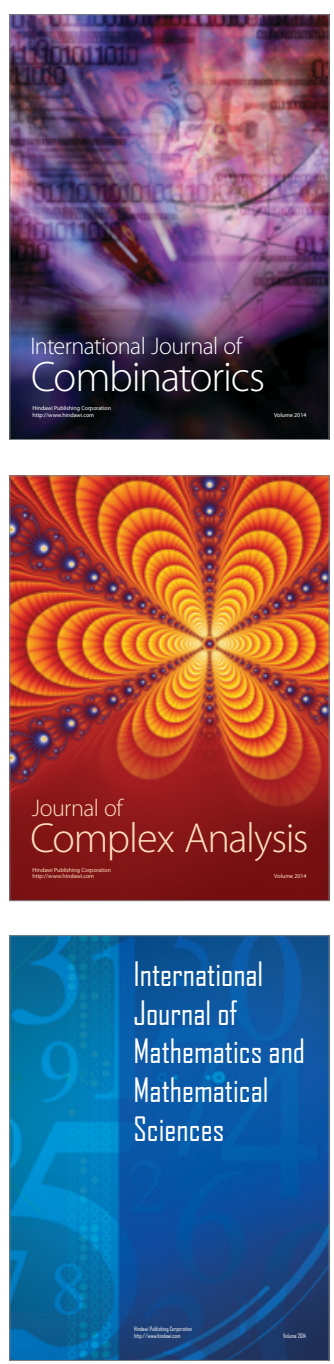
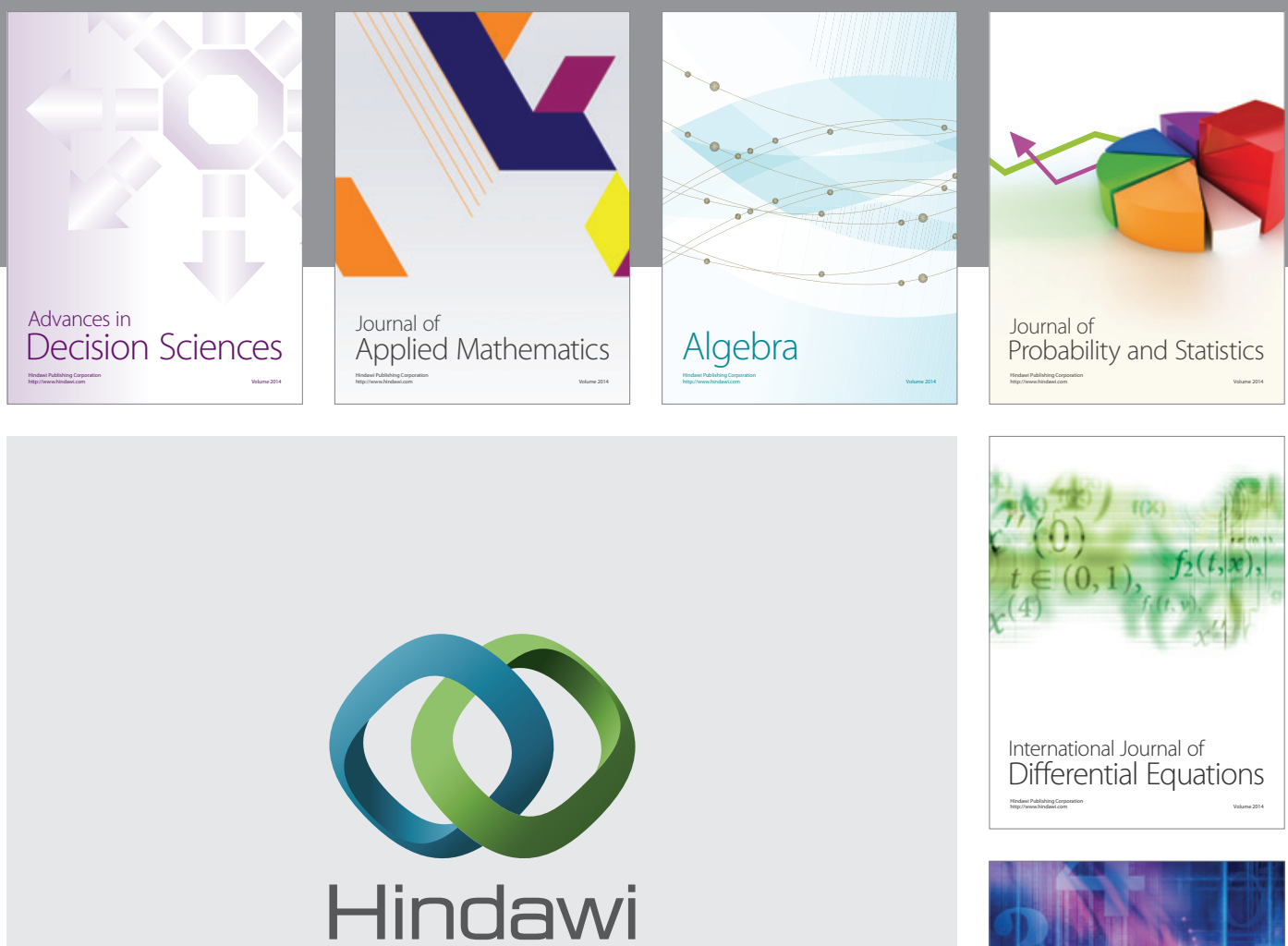

Submit your manuscripts at http://www.hindawi.com
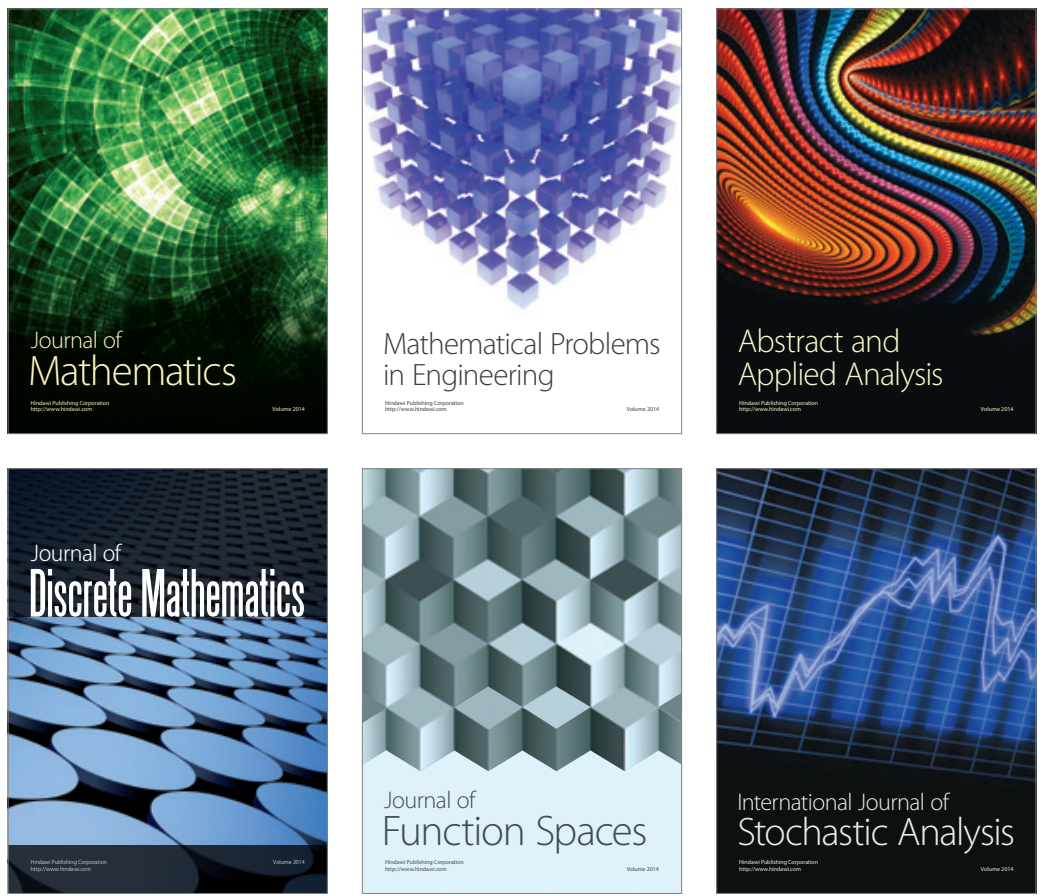

Journal of

Function Spaces

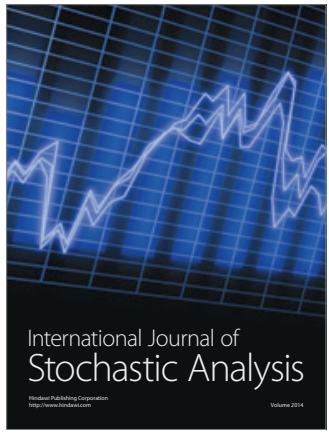

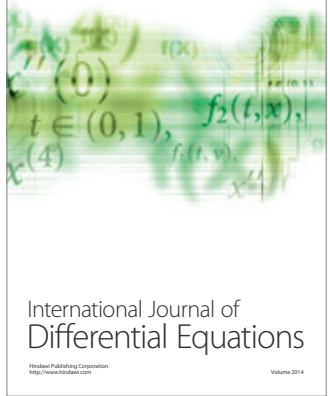
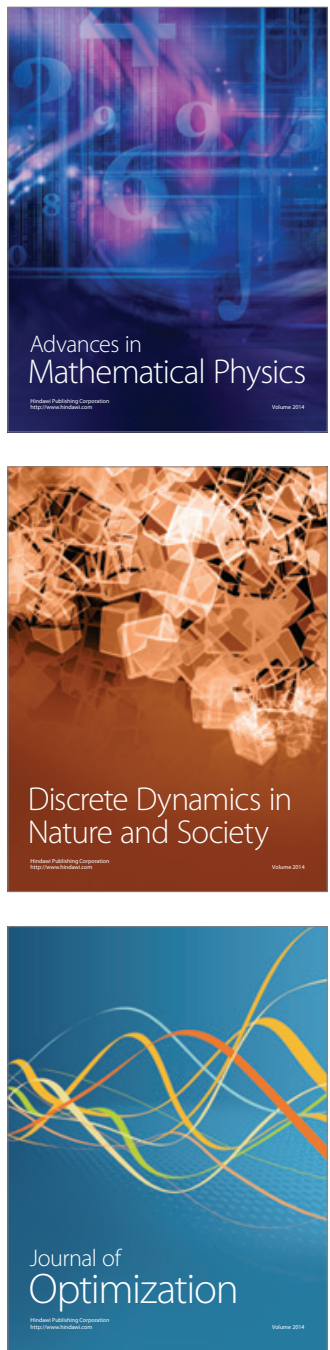